\title{
Ubiquitous Experience Media
}

\author{
Kenji Mase \\ Graduate School of Information Science, Nagoya University, Japan \\ mase@nagoya-u.jp
}

\begin{abstract}
Our daily activity in the future society will be facilitated by recording personal and joint experiences in the real and cyber world with a ubiquitous computing environment. We can exploit the recorded activity as a good source of human-human and human-computer communication for sharing experience, memory and knowledge. We are interested in audio-visual, ubiquitous, and/or wearable experience-capturing technology as an interaction-grounded lifelog tool. The talk addresses the advanced technologies of non-cumbersome indexedrecording, abstracting, and summarizing of experiences as well as sharing these experiences among people. We have developed several devices, algorithms and tools, such as a wearable interaction tracker and a multipoint-view Peg-Scope Viewer, which facilitate indexing the personal and joint experiences. Indexing experiences is a key technology for realizing usable future computational media, which we call ubiquitous experience media (UEM).
\end{abstract}

Keywords: Ubiquitous computing; personal experience. 